\section{A comparison of microscopic examination and rapid diagnostic tests used in Guyana to diagnose malaria}

\author{
Rajini Kurup, Rena Marks \\ Faculty of Health Sciences, University \\ of Guyana, Georgetown, Guyana, \\ South America
}

\section{Abstract}

The aim of this study was to compare rapid diagnostic tests (RDTs) for malaria with routine microscopy for a prompt and accurate diagnosis of malaria and to provide an effective disease management in Guyana. Blood samples were collected randomly from 624 patients with clinical suspicion of malaria from four private hospitals in Georgetown, Guyana. The five different test methods [Paramax-3, Optimal-IT, VISITECT Malaria Combo PAN/Pf, Standard Diagnostic (SD) Bioline and conventional Giemsa stain microscopy] were performed independently by well trained and competent laboratory staff to assess the presence of malaria parasites. Results from the rapid diagnostic kits were analyzed and compared to those obtained by general microscopy. Of the 624 patients involved in the study, 197 (31.6\%) tested positive and 427 (68.4\%) tested negative to RDT whereas $190(30.4 \%)$ tested positive and 434 (69.6\%) tested negative to microscopy. The positive agreement index between RDT and microscopy was 89\%. A comparison of microscopy with the RDTs, Paramax, OpitmalIT, Omega, SD, showed a positive agreement index of $93 \%, 86 \%, 80 \%$ and $86 \%$, respectively. The study, therefore, highlights the importance of both methods in diagnosis of malaria in endemic areas. Microscopy is the more reliable method in rural areas where malaria is most prevalent. RDT offers a good alternative, being an easy and rapid method that does not require an experienced laboratory technician.

\section{Introduction}

Malaria is an extremely complex disease that has been responsible for deaths and social disruption since the beginning of recorded human history. A large number of suspected malaria cases are still not suitably identified, resulting in the overuse of anti-malarial drugs and poor disease monitoring. ${ }^{1-3}$ The World Health Organization has recommended that management of all malaria cases should be confirmed by quality-assured, parasite-based diagnosis before treatment is started. ${ }^{3-4}$ Parasite densities of around 200 parasites per microliter (parasites/L) should be detected to ensure high field sensitivity for clinically significant malaria infection in many malaria endemic populations. ${ }^{5}$ High sensitivity of malaria diagnosis is important in all settings, and is essential for the most vulnerable population groups. This is particularly important for infants and pregnant women who are put at greater risk by a wrong diagnosis. In these subjects, malaria infection produces an acute illness that can rapidly progress to death..$^{6-7}$

Malaria remains a very serious health problem in Guyana, South America. It affects mainly the indigenous population living in rural/hinterland communities and miners working in those areas. Malaria is not considered to be the major cause of death overall in Guyana but becomes a great threat when combined with malnutrition or if repeated episodes are experienced. According to available data, cases of malaria are on the increase, with the majority occurring in inland regions. ${ }^{8}$

Currently, microscopic examination of thin or thick blood smears is widely used for diagnosis of malaria. Giemsa microscopy is regarded as the most suitable diagnostic instrument for malaria control because it is inexpensive to perform, and it is able to differentiate malaria species and quantify parasites. ${ }^{9}$ At low level parasitemia, the sensitivity of microscopy is limited and the method is time consuming, labor intensive, and requires an experienced microscopist. ${ }^{10}$ The sensitivity of various diagnostic methods also depends on the malaria species, the parasite density, previous treatment, gametocytemia and quality of the diagnostic method. ${ }^{11}$

The national malaria control programs of several countries have been using rapid diagnostic tests (RDTs) as a definitive diagnostic tool where microscopy is not readily available to confirm suspected malaria cases. ${ }^{12}$ However, in Guyana, most of these RDTs are used in the private sector.

This study, therefore, explores the importance of microcopy and different diagnostic methods for the detection of Plasmodium species in Guyana.

\section{Materials and Methods}

This prospective study was conducted in Georgetown (Region n. 4), an urban region of Guyana, between July and October 2011. Samples were obtained from one private laboratory (Laboratory $\mathrm{A}$ ), one private clinic (Laboratory B), and two private hospitals (Laboratory $\mathrm{C}$ and Laboratory D) in the city of Georgetown, Guyana. These private institu-
Correspondence: Rajini Kurup, Faculty of Health Sciences, University of Guyana, South America. E-mail: kuruprajini@yahoo.com

Key words: rapid diagnostic tests, malaria rapid diagnostic devices, microscopy.

Acknowledgements: the authors would like to thank all the laboratory staff who participated in this study for their co-operation during the research.

Contributions: RK, conception and design, analysis and interpretation of data as well as revising and final approval of the version to be published; RM, collecting samples for diagnosing and drafting the article

Conflict of interests: the authors declare no potential conflict of interests.

Received for publication: 27 October 2011. Revision received: 2 May 2012.

Accepted for publication: 2 May 2012

This work is licensed under a Creative Commons Attribution NonCommercial 3.0 License (CC BYNC 3.0).

(C) Copyright R. Kurup and R. Marks, 2012

Licensee PAGEPress, Italy

Malaria Reports 2012; 2:e2

doi:10.4081/malaria.2012.e2

tions use RDTs as their main diagnostic tool for malaria. However, Laboratory B would follow up with microscopy only if the RDT result is positive.

All tests were carried out immediately and examined by well trained and competent laboratory staff. For microscopy, the test was examined independently by expert microscopists in different settings who did not know the results of RDT. Written results were communicated immediately to the clinicians.

\section{Eligibility for participation}

All participants were patients who presented with signs and symptoms of a malaria infection and for whom a malaria test was requested by a physician. Those who had recently returned from an endemic rural region of Guyana with a high prevalence of malaria were also suitable candidates for this investigation. A total of 624 samples were obtained.

\section{Rapid diagnostic tests}

Four different brands/types of RDT were used by the different laboratories: Paramax-3, OptiMal-IT, VISITECT Malaria Combo PAN/Pf, and Standard Diagnostic Bioline, Malaria Ag $\mathrm{Pf} / \mathrm{Pv}$ (SD). Laboratory A used SD (batch $\mathrm{n}$. 018110); Laboratory B used VISITECT Malaria Combo PAN/Pf (batch ns. 126013, 126041 and 
126043) and 0ptiMal-IT (batch n. 0E0015M); Laboratory C used Paramax-3 (batch ns. 9117 and 9121), and Laboratory D used OptiMal-IT (batch ns. 0K0027M and 0E0015M) and Paramax-3 (batch ns. 9111 and 9114).

\section{Paramax-3 (rapid test for malaria pan/P. vivax/P. falciparum)}

This is a rapid, self-testing, qualitative, 2-site sandwich immunoassay utilizing whole blood to detect Plasmodium falciparum specific histidine rich protein-2 (Pf HRP-2), Plasmodium vivax specific parasite lactase dehydrogenase (pLDH) and pan malaria specific pLDH. This test can be used for the specific detection of $P$. falciparum and $P$. vivax malaria, and to differentiate other malarial species.

\section{OptiMal-IT}

DiaMed OptiMal-IT is an immune-chromatography test using monoclonal antibodies against the metabolic enzyme pLDH of Plasmodium spp. These monoclonal antibodies are classified in two groups: i. specific for $P$. falciparum; ii. using pan-specific monoclonal antibodies reacting with all four species of Plasmodium spp. that can occur in human beings: $P$. falciparum, $P$. vivax, $P$. ovale, and $P$. malariae.

It is specific for the detection of $\mathrm{pLDH}$, an enzyme produced by both sexual and asexual forms of the parasites and sensitive for the detection of peripheral parasitemia levels of $0.001-0.002 \%$ (50-100 parasites/ L of blood).

\section{VISITECT Malaria Combo PAN/}

\section{P. falciparum}

VISITECT Malaria Combo Pan/Pf is a rapid test for the detection of $P$. falciparum, non- $P$. falciparum or mixed infections that utilizes the principle of immune-chromatography. This kit determines malarial infection by the detection of pan malaria specific pLDH released from parasitized red blood cells. Additionally, it determines the specific infection of $P$. falciparum by the detection of $P$. falciparum specific Pf HRP-2, a water soluble protein that is released from parasitized erythrocytes of infected individuals and is species specific.

\section{SD Bioline Malaria Ag $P$. vivax/P. falciparum}

The SD Bioline Malaria Pf/Pv test is a rapid immunochromatographic test for qualitative detection of antibodies of all isotypes (IgG, IgM, IgA) specific to $P$. falciparum and $P$. vivax simultaneously in human whole blood. Whole blood can be used for testing immediately or stored at $2-8^{\circ} \mathrm{C}$ for up to three days. This test is intended for initial screening and all positive specimens should be confirmed by microscopic examination.

\section{Microscopy diagnosis}

All of the samples obtained were first tested by RDTs, then the same samples were used to make thick and thin smears. A small number of smears were made from fresh capillary blood. The remaining smears were made from venous blood obtained from the patient added to an EDTA collection tube. These were stored at $2-8^{\circ} \mathrm{C}$ for between 24 to $48 \mathrm{~h}$ after being tested by RDT and prior to microscopic examination. The smears were processed by fixing the thin film in absolute methanol (methyl alcohol), heat fixed and stained with $10 \%$ Giemsa solution in buffered water, $\mathrm{pH} 7.2$ for $10-12$ min. After staining, the smears were rinsed with normal water, drained and air dried. They were then examined by light microscopy under 1000x magnification for malaria parasites, Plasmodium species and quantization. A malaria blood film was considered negative after 100 high power fields had been examined and no parasite observed. The parasite count per microliter of blood was obtained by using the formula: [parasite count/200 white blood cell $(\mathrm{WBC})] \times$ absolute WBC count. ${ }^{13}$ If the parasite density was found to be more than 100 parasites/field in a thick film, the thin film was used for the count. Upon the observation of asexual malaria parasites, parasitized red blood cells (RBCs) were counted against 1000 RBCs. The parasite count per microliter of blood was obtained using the formula: (parasite count/1000 RBC) $\times$ absolute RBC count. ${ }^{14}$ The thin film was used for species identification of detected malaria parasites.

\section{Ethical approval}

Ethical approval for this research was granted by the Ethical Review Committee of the Ministry of Health, Georgetown, Guyana, South America.

Table 1. Performance of different rapid diagnostic tests methods compared to Giemsa stain microscopy.

\begin{tabular}{|c|c|c|c|c|c|c|c|}
\hline \multirow[t]{2}{*}{ RTDs } & \multicolumn{2}{|c|}{ Microscopy } & \multirow{2}{*}{$\begin{array}{c}\text { Sensitivity (\%) } \\
\text { (95\% CI) }\end{array}$} & \multirow{2}{*}{$\begin{array}{c}\text { Specificity (\%) } \\
\text { (95\% CI) }\end{array}$} & \multirow[t]{2}{*}{ PPV (\%) } & \multirow[t]{2}{*}{ NPV (\%) } & \multirow[t]{2}{*}{ Accuracy } \\
\hline & Positive & Negative & & & & & \\
\hline Positive & 172 & 25 & $90.5(86.6-93.5)$ & $94.2(92.5-95.6)$ & 87.3 & 95.8 & 93.1 \\
\hline Negative & 18 & 409 & & & & & \\
\hline
\end{tabular}

Mc Nemar test $=0.36 ; \mathrm{P}<0.05 ;$ Accuracy=93.3\%; kappa=83.9. RTDs, rapid diagnostic tests; PPV, positive predictive value; NPV, negative predictive value.

Table 2. Diagnostic performances of Paramax, OptiMAL-IT, Omega and SD tests compared to that of microscopy.

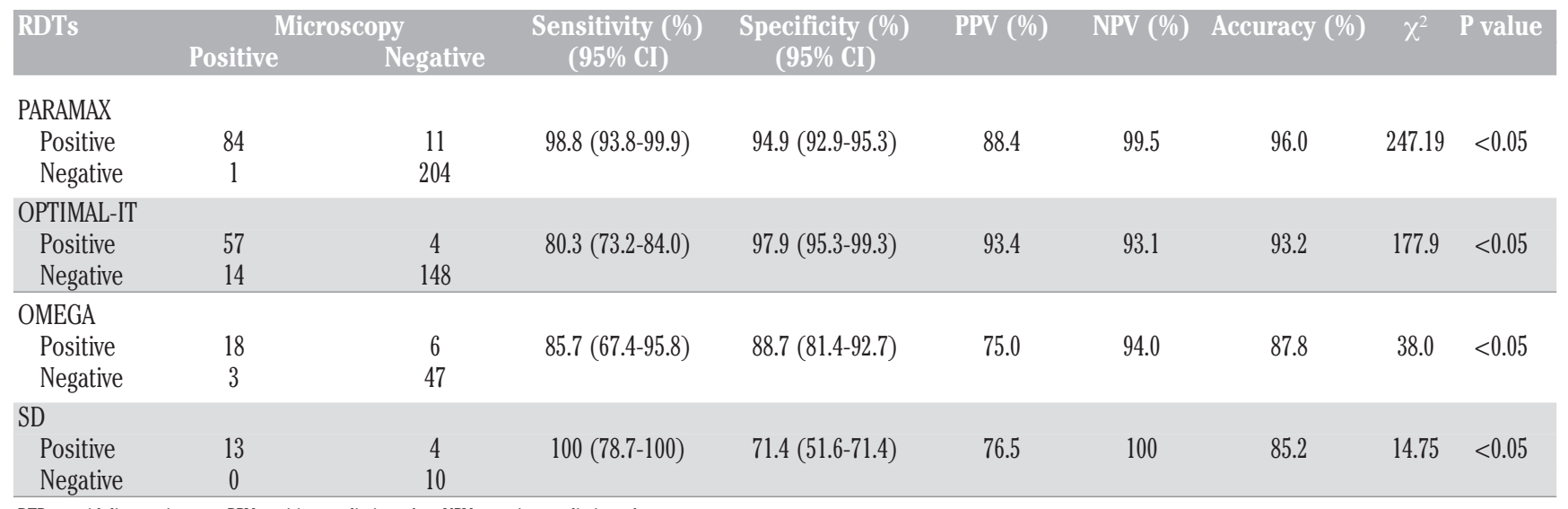

RTDs, rapid diagnostic tests; PPV, positive predictive value; NPV, negative predictive value. 


\section{Statistical analysis}

Since none of the diagnostic methods tested in this study is considered as gold standard, statistical analysis was carried out according to Erhart et al., Bhattarai et al. and Speybroeck et al. ${ }^{15-17}$ The agreement between the two tests was evaluated by calculating positive and negative agreement indices according to the theory proposed by Graham and Bull. ${ }^{18}$ Considering the readings of the two tests reported as either positive or negative, the values $a, b, c$ and $d$ denote the observed frequencies for each possible combination of ratings by tests 1 and 2 .

a. the number of samples positive with both

tests;

b. the number of samples negative with test 1 and positive with test 2 ;

c. the number of samples positive with test 1 and negative with test 2 ; and

d. the number of samples negative with both tests.

The proportion of specific agreement for the overall agreement $\left(\mathrm{P}_{0}\right)$, the positive ratings $\left(\mathrm{P}_{\mathrm{pos}}\right)$ (the positive agreement index), and for the negative ratings $\left(\mathrm{P}_{\text {neg }}\right)$ (the negative agreement index) were calculated as follows:

$$
\begin{aligned}
& P_{0}=(a+d) / \text { total } \\
& P_{\text {pos }}=2 \mathrm{a} / 2 \mathrm{a}+\mathrm{b}+\mathrm{c} \\
& \mathrm{P}_{\text {neg }}=2 \mathrm{~d} / 2 \mathrm{~d}+\mathrm{b}+\mathrm{c}
\end{aligned}
$$

This method excludes the limitation of the kappa statistics. Mean, standard deviation, prevalence and confidence intervals were calculated using SPSS 11 software. The calculations of positive and negative agreement indices and overall agreement indices were made according to Erhart et al., Bhattarai et al. and Speybroeck et al. ${ }^{15-17}$

\section{Results}

A total of 624 patients, aged 1-83 years (mean 33.6 years \pm 14.4 years) suspected of malaria or presenting a history suggestive of malaria were included in the study. The study involved more males 443 (71\%) than females 181 (29\%).

\section{Giemsa stain microscopy}

The results of parasite detection by microscopy are shown in Tables 1 and 2 . Diagnostic performances of the tests with their batch numbers are shown in Table 3 . The Giemsa stain microscopy test reported 190 slide positive cases of malaria (30.5\%). Positivity with RDTs was $31.6 \%$. Out of these positive films, 163 slides (85.8\%) were positive with mono-infection. Species determination identified 99 (52\%) slides with mono-infection of $P$. falciparum (Pf), 63 (33\%) slides with mono-infection of $P$. vivax, and one (0.5\%) slide with mono-infection $P$. malariae $(\mathrm{Pm})$. Mixed infections were recorded in 27 (14.2\%) slides (Table 4).

Microscopy recorded parasite count with $P$. falciparum ranging from 24 to 105,600 parasites/ $\mu \mathrm{L}$ (mean count 11,002.4 parasites/ $\mu \mathrm{L}), P$. vivax ranging from 96 to 163,800 parasites/ $\mu \mathrm{L}$ (mean count 12,736.3 parasites/ $\mu \mathrm{L}$ ), P. malariae with mean count 1469 parasites/ $\mu \mathrm{L}$ and $P$. falciparum gametocyte recorded 24 to 12,330

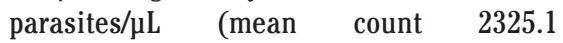
parasites/ $\mu \mathrm{L})$.

\section{Rapid diagnostic tests}

\section{Paramax}

Of the total 300 patients tested for malaria with Paramax against microscopy, 95 (31.6\%) were Paramax positive compared to 85 (28.3\%) positive by microscopy: 59 cases were $P$. falciparum positive, 28 cases were $P$. vivax positive, and 8 cases were both $P$. falciparum and $P$. vivax positive.

\section{OptiMAL-IT}

Of the 223 patients tested for malaria with OptiMal-IT against microscopy, according to OptiMAL-IT testing, 35 cases were $P$. falciparum positive, 23 cases were $P$. vivax and 3 cases both $P$. falciparum and $P$. vivax positive (Table 4).

\section{VisiTect}

Overall, 74 patients were tested for malaria with VisiTect against microscopy: 7 were $P$. falciparum positive, $7 P$. vivax positive, and 10 cases both $P$. falciparum and $P$. vivax positive.

\section{Standard Diagnostic}

Of the 27 patients tested for malaria with SD against microscopy, 6 cases were $P$. falciparum positive, 5 cases were $P$. vivax positive and 6 cases both $P$. falciparum and $P$. vivax positive.

\section{Concurrence between microscopy and rapid diagnostic tests}

Comparison between microscopy and RDTs were assessed by calculating positive and negative agreement indices. Table 2 shows positive and negative agreement index among different RDT kits. A high positive agreement index was observed in all RDTs except SD.

\section{Discussion}

Malaria can be a life-threatening disease in a vulnerable population if not treated. Therefore, a quick and accurate diagnosis is very important. To prevent unnecessary antimalarial treatments, it is important to confirm clinical suspicions with a good laboratory test. RDTs for malaria are being increasingly adopted across endemic countries to strengthen parasitological diagnosis and appropriate management of all cases of fever. They are particularly valuable in areas which do not have good resources for microscopy.

Most RDTs only record the presence or absence of antigens but cannot measure the parasite density. These should, therefore, only be considered as an extended means of parasite based diagnosis where microscopy is absent due to its varied diagnostic applications and importance of supportive patient management. ${ }^{4,19-21}$ The national malaria control programs of several countries have been using these RDTs as a definitive diagnostic tool where microscopy is not readily available to confirm suspected malaria cases. ${ }^{12}$ However, in Guyana most of these RDTs are used in the private sector.

In this study, we assessed the field performance of the RDT among different hospital laboratory and private laboratories using conventional Giemsa stain thick and thin blood films. In the current study, more infections were detected by RDT than by blood slide microscopy.

Among all the four RDTs used in this study, OptiMAL had the lowest positive results compared to microscopy. This could be because some malaria infections detected by blood films were not detected by the OptiMAL test because the latter detects pLDH which is produced only by living parasites. It is possible that some of the patients infected with malaria medicated themselves when malaria symptoms appeared during this outbreak and did not report this to the attending clinician. Self treatment of malaria is widespread in Guyana (Kurup and Kumar; unpublished results, 2011). There are other possible explanations for discrepancies in test results obtained by blood film examination and by the OptiMAL test, including: i) insufficient detection of low parasitemia levels by OptiMAL; ii) the sequestration of parasites; and iii) false-positive reactions.

In areas where there is a high incidence of malaria, the lack of facilities undermines the benefits of RDTs. RDTs, however, are sensitive diagnostic tools for malaria. They are also simple to use and provide quick results without the need for good microscopic equipment and electricity, making them a good alternative to microscopy in endemic areas. ${ }^{22}$

Marx and others, ${ }^{23}$ in their systematic review on the accuracy of RDT for malaria in returning travelers, indicates that, despite a low sensitivity, RDT will lead to the detection of most clinically relevant $P$. falciparum cases with considerably better accuracy than that to be expected from routine microscopy. The current study confirms that RDT in conjunction 
with microscopy should improve the diagnosis of malaria. However, RDT use should be considered as more cost-effective in the areas characterized by high-moderate intensity malaria transmission and in situations where health services are inadequate or absent. ${ }^{23}$

On other hand, RDTs only record the presence or absence of antigens but cannot measure the parasite density. They should, therefore, only be considered to be an extended means of parasite based diagnosis where microscopy is absent due to its varied diagnostic applications and the importance of supportive patient management. ${ }^{4,19-21}$

Reports from elsewhere indicate that RDTs have shown a comparable level of accuracy to microscopy in clinical settings. ${ }^{24,25}$ Even though the clinical history of the participants was not recorded in our study, evidence from other studies showed that RDT positive cases missed by microscopy might be individuals who had been treated but in whom antigenemia persists. ${ }^{25,26} \mathrm{~A}$ potential alternative explanation for this level of false positives is sequestration: erythrocytes containing mature parasites clump together in the microvasculature and are, therefore, not seen in the peripheral circulation and blood films, while antigen continues to be released. ${ }^{27}$ It may also be possible that the parasite density was too low to be

Table 3. Diagnostic performances of individual rapid diagnostic tests and their Batch number compared to that of microscopy.

\begin{tabular}{|c|c|c|c|c|c|c|c|c|c|}
\hline \multirow[t]{2}{*}{ RDTs } & \multicolumn{2}{|c|}{ Microscopy } & \multirow{2}{*}{$\begin{array}{c}\text { Sensitivity (\%) } \\
(95 \% \mathrm{CI})\end{array}$} & \multirow{2}{*}{$\begin{array}{l}\text { Specificity (\%) } \\
(95 \% \text { CI) }\end{array}$} & \multirow[t]{2}{*}{ PPV (\%) } & \multirow[t]{2}{*}{ NPV (\%) } & \multirow[t]{2}{*}{ Accuracy (\%) } & \multirow[t]{2}{*}{$x^{2}$} & \multirow[t]{2}{*}{ P value } \\
\hline & Positive & Negative & & & & & & & \\
\hline \multicolumn{10}{|l|}{ PARAMAX } \\
\hline \multicolumn{10}{|l|}{ Batch 91111} \\
\hline Positive & 16 & 8 & $96.9(86.4-99.8)$ & $97.0(91.9-98.4)$ & 93.9 & 98.5 & 96.9 & 85.0 & $<0.05$ \\
\hline Negative & 0 & 66 & & & & & & & \\
\hline \multicolumn{10}{|l|}{ Batch 91114} \\
\hline Positive & 31 & 2 & $100(79.3-100)$ & $89.2(84.7-89.2)$ & 66.7 & 100 & 91.9 & 53.5 & $<0.05$ \\
\hline Negative & 1 & 64 & & & & & & & \\
\hline \multicolumn{10}{|l|}{ Batch 91117} \\
\hline Positive & 27 & 1 & $100(89.2-100)$ & $98.1(92.6-98.1)$ & 96.4 & 100 & 98.8 & 75.7 & $<0.05$ \\
\hline Negative & 0 & 52 & & & & & & & \\
\hline \multicolumn{10}{|l|}{ Batch 91121} \\
\hline Positive & 10 & 0 & $100(75.2-100)$ & $100(88.8-100)$ & 100 & 100 & 100 & 32.0 & $<0.05$ \\
\hline Negative & 0 & 22 & & & & & & & \\
\hline \multirow{2}{*}{\multicolumn{10}{|c|}{$\begin{array}{l}\text { OPTIMAL - IT } \\
\text { Batch 0E0015M }\end{array}$}} \\
\hline & & & & & & & & & \\
\hline Positive & 42 & 4 & $84.0(74.9-89.1)$ & $96.0(91.5-98.6)$ & 91.3 & 92.3 & 92 & 100.3 & $<0.05$ \\
\hline Negative & 8 & 96 & & & & & & & \\
\hline \multicolumn{10}{|c|}{ Batch 0K0027M } \\
\hline Positive & 15 & 0 & $71.4(56.2-71.4)$ & $100(93.8-100)$ & 100 & 100 & 91.8 & 46.7 & $<0.05$ \\
\hline Negative & 6 & 52 & & & & & & & \\
\hline \multirow{2}{*}{\multicolumn{10}{|c|}{ OMEGA }} \\
\hline \multicolumn{9}{|l|}{ Batch 126013} & \\
\hline Positive & 4 & 4 & $80(33.3-98.9)$ & $78.9(66.6-83.9)$ & 50.0 & 93.8 & 79.2 & 6.2 & $<0.05$ \\
\hline Negative & 1 & 15 & & & & & & & \\
\hline \multicolumn{10}{|c|}{ Batch 206041} \\
\hline Positive & 8 & 1 & $80(51.8-89.5)$ & $95.7(83.4-99.8)$ & 88.9 & 91.7 & 90.9 & 20.1 & $<0.05$ \\
\hline Negative & 2 & 21 & & & & & & & \\
\hline \multicolumn{10}{|l|}{ Batch 206043} \\
\hline Positive & 6 & 1 & $100(60.7-100)$ & $91.7(72.0-91.7)$ & 85.7 & 100 & 94.4 & 14.1 & $<0.05$ \\
\hline Negative & 0 & 11 & & & & & & & \\
\hline \multicolumn{10}{|l|}{ SD } \\
\hline \multicolumn{10}{|l|}{ Batch 018110} \\
\hline Positive & 13 & 4 & $100(78.7-100)$ & $71.4(51.6-71.4)$ & 76.5 & 100 & 85.2 & 14.7 & $<0.05$ \\
\hline Negative & 0 & 10 & & & & & & & \\
\hline
\end{tabular}

RTDs, rapid diagnostic tests; PPV, positive predictive value; NPV, negative predictive value.

Table 4. Comparison of slide microscopy and rapid diagnostic tests by species detected.

\begin{tabular}{|c|c|c|c|c|c|c|c|c|c|}
\hline RDTs & Neg & $\mathrm{Pf}$ & Pfm & $\begin{array}{l}\text { Microscopy } \\
\text { Pfv }\end{array}$ & Pfvm & $\mathrm{Pm}$ & $\mathrm{Pv}$ & Pvm & Total \\
\hline Neg & $409(65.5)$ & $14(2.2)$ & 0.0 & 0.0 & 0.0 & 0.0 & $4(0.6)$ & 0.0 & $427(68.4)$ \\
\hline $\mathrm{Pf}$ & $19(3.0)$ & 78 (12.5) & 0.0 & $4(0.6)$ & $2(0.3)$ & 0.0 & $4(0.6)$ & 0.0 & $107(17.1)$ \\
\hline $\mathrm{Pfv}$ & $1(0.2)$ & $6(1.0)$ & $3(0.5)$ & $14(2.2)$ & 0.0 & 0.0 & $3(0.5)$ & 0.0 & $27(4.3)$ \\
\hline $\mathrm{PV}$ & $5(0.8)$ & $1(0.2)$ & 0.0 & $3(0.5)$ & 0.0 & $1(0.2)$ & $52(8.3)$ & $1(0.2)$ & $63(10.1)$ \\
\hline Total & $434(69.6)$ & 99 (15.9) & $3(0.5)$ & $21(3.4)$ & $2(0.3)$ & $1(0.2)$ & $63(10.1)$ & $1(0.2)$ & 624 \\
\hline
\end{tabular}

RTDs, rapid diagnostic tests. Pf, P. falciparum; Pfm, P. falciparum \& P. malariae; Pfv, P. falciparum \& P. vivax; Pfvm, P. falciparum, P. vivax \& P. malariae; Pm, P. malariae; Pv, P. vivax; Pvm, P. vivax \& P. malariae. 
seen by microscopy but that there was sufficient parasite antigen to result in a positive RDT. ${ }^{28}$

Although molecular tests such as RDT should be preferred to microscopy, RDT testing for confirmation of malaria can not be used in countries like Guyana since the protocols are too cumbersome, too expensive, and are not simple or rapid, or even not available at all, because of limited resources such as a lack of electricity and inadequate laboratory infrastructure. ${ }^{29}$

In this study, both RDT and microscopy provided comparable results. Therefore, RDT in conjunction with microscopy should be used to improve the diagnosis of malaria. RDT use should be considered more cost-effective in the areas characterized by high-moderate intensity malaria transmission and in situations where health services are inadequate or absent. $^{30}$

\section{References}

1. Greenwood B, Mutabingwa T. Malaria in 2002. Nature 2002;415:670-2.

2. Snow RW, Guerra CA, Noor AM, et al. The global distribution of clinical episodes of Plasmodium falciparum malaria. Nature 2005;434:214-7.

3. World Health Organization. Malaria rapid diagnostic test performance; results of WHO product testing of malaria RDTs: Round 2. Geneva: World Health Organization; 2009. http://apps.who.int/..J tdr-research-publications/rdt_round2 Accessed: March 19, 2011.

4. World Health Organization. Parasitological confirmation of malaria diagnosis. Geneva: World Health Organization; 2009. http://whqlibdoc.who.int/publications/2010/9789241599412_eng.pdf Accessed: March 1, 2011.

5. World Health Organization. Towards quality testing of malaria rapid diagnostic tests. Evidence and methods. Geneva: World Health Organization; 2006. http://www.who.int/entity/malaria/publications/atoz/929061238X/en/index.html Accessed: March 6, 2011.

6. World Health Organization. The role of laboratory diagnosis to support malaria disease management - focus on the use of rapid diagnostic tests in areas of high transmission. Geneva: World Health Organization; 2006. http://helid.digicollection.org/en/d/Js13417e/6.html Accessed: January 4, 2011.
7. Greenwood BM. The syndromic approach to malaria diagnosis. Draft position paper prepared for informal consultation on malaria diagnostics at the turn of the century. Geneva: World Health Organization; 1999. (WHO/CDS/IC/WP 99.2).

8. United Nations Development Programme. The Millennium Development Goals 2005 Goal 6: Combat HIV/AIDS, malaria and other diseases.http://www.undp.org.gy/ web/index.php?option=com_content\&vie $\mathrm{w}=$ article \&id=69\&Itemid $=71$ Accessed: February 27, 2011.

9. Wongsrichanalai C, Barcus MJ, Muth S, et al. A review of malaria diagnostic tools: Microscopy and Rapid Diagnostic Tests (RDT). Am J Trop Med Hyg 2007;77.119-27.

10. Dourado HN, Abdon NP, Martins SJ. Falciparum malaria. Infect Dis Clin N Am 1994;8:207-23.

11. Arora S, Gaiha M, Arora A. Role of the Parasight-F test in the diagnosis of complicated Plasmodium falciparum malarial infection. Braz J Infect Dis 2003;7:332-8.

12. Forney JR, Wongsrichanalai C, Magill AJ, et al. Devices for rapid diagnosis of Malaria: evaluation of prototype assays that detect Plasmodium falciparum histidine-rich protein 2 and a Plasmodium vivax-specific antigen. J Clin Microbiol 2003;41:2358-66.

13. Jeremiah ZA, Uko EK. Comparative analysis of malaria parasite density using actual and assumed white blood cell counts. Ann Trop Ped 2007;27:75-9.

14. Frean JA. Reliable enumeration of malaria parasites in thick blood films using digital image analysis. Malaria J 2009;8:218.

15. Erhart A, Dorny P, Van De N, et al. Taenia solium cysticercosis in a village in northern Viet Nam: seroprevalence study using an ELISA for detecting circulating antigen. Trans R Soc Trop Med Hyg 2002;96:270-2.

16. Bhattarai NR, Van der Auwera G, Khanal $\mathrm{B}$, et al. PCR and direct agglutination as Leishmania infection markers among healthy Nepalese subjects living in areas endemic for Kala-Azar. Trop Med Int Health 2009;14:404-11.

17. Speybroeck N, Praet N, Claes F, et al. True versus apparent malaria infection prevalence: the contribution of a Bayesian approach. PLoS 2011;6:e16705.

18. Graham P, Bull B. Approximate standard errors and confidence intervals for the indices of positive and negative agreement. J Clin Epidemiol 1998;51:763-71.

19. Kolaczinski J, Mohammed N, Ali I, et al. Comparison of the OptiMAL rapid antigen test with field microscopy for the detection of Plasmodium vivax and P. falciparum: considerations for the application of the rapid test in Afghanistan. Ann Trop Med Parasitol 2004;98:15-20.

20. Stephens J, Phanart K, Rooney W, Barnish G. A comparison of three malaria diagnostic tests, under field conditions in northwest Thailand. Southeast Asian J Trop Med Public Health 1999;30:625-30.

21. Wongsrichanalai C, Barcus MJ, Muth S, et al. A review of malaria diagnostic tools: microscopy and rapid diagnostic test (RDT). Am J Trop Med Hyg 2007;77:119-27.

22. Mboera L, Fanello C, Malima R, et al. Comparison of the Paracheck-Pf rest with microscopy, for the confirmation of Plasmodium falciparum malaria in Tanzania. Ann Trop Med Parasitol 2006; 100:115-22.

23. Marx A, Pewsner D, Egger M, et al. Metaanalysis: accuracy of rapid tests for malaria in travellers returning from endemic areas. Ann Int Med 2005;142:836-46.

24. Moonasar D, Goga AE, Frean J, et al. An exploratory study of factors that affect the performance and usage of rapid diagnostic tests for malaria in the Limpopo Province, South Africa. Malaria J 2007;6:74.

25. Moody A. Rapid diagnostic tests for malaria parasites. Clin Microbiol Rev 2002;15: 66-78.

26. World Health Organization. The role of laboratory diagnosis to support malaria disease management: Focus on the use of rapid diagnostic tests in areas of high transmission, report of a WHO technical consultation, 25-26 October 2004. Geneva: World Health Organization; 2008. pp 4-48.

27. Dondorp AM, Desakorn V, Pongtavor npinyo W, et al. Estimation of the total parasite biomass in acute falciparum malaria from plasma PfHRP2. PLoS Med 2005; 2:e204.

28. Bell DR, Wilson DW, Martin LB. False-positive results of a Plasmodium falciparum histidine-rich protein 2-detecting malaria rapid diagnostic test due to high sensitivity in a community with fluctuating low parasite density. Am J Trop Med Hyg 2005;73:199-203.

29. Hanscheid T, Grobusch MP. How useful is PCR in the diagnosis of malaria? Trends Parasitol 2002;18:395-8.

30. Nicastri E, Bevilacqua N, Schepisi MS, et al. Accuracy of Malaria diagnosis by microscopy, rapid diagnostic test, and PCR methods and evidence of antimalarial overprescription in non-severe febrile patients in two Tanzanian hospitals. Am J Trop Med Hyg 2009;80: 712-7. 